\title{
FUSE Observations of the Central Star of Abell 78
}

\author{
Klaus Werner, Stefan Dreizler \\ Institut für Astronomie und Astrophysik, Univ. Tübingen, Germany
}

Lars Koesterke

NASA/GSFC, Greenbelt MD, U.S.A.

Jeff W. Kruk

Department of Physics and Astronomy, JHU, Baltimore MD, U.S.A.

\begin{abstract}
FUSE high resolution spectra of two PG1159 type central stars (K1-16 and NGC 7094) have revealed an unexpected iron deficiency of at least 1 or 2 dex (Miksa et al. 2002). Here we present early results of FUSE spectroscopy of the CSPN Abell 78. It is shown that iron is strongly deficient in this star, too.
\end{abstract}

The existence of hydrogen-deficient central stars (spectral types PG1159 and [WC]) is probably due to a late thermal pulse. PG1159 central stars are very hot $\left(T_{\text {eff }}>100000 \mathrm{~K}\right)$ and metals are highly ionized. The dominant ionization stage of iron in the line formation region is Fe VII and most of its lines are located in the FUV region not accessible by HST. The lines are expected to be rather weak and narrow. Due to the faintness of PG1159 stars, iron abundances could not be determined until the advent of FUSE. It was expected that the iron abundance would be essentially solar, because iron group elements do not participate in any charged particle reactions occurring in low- and intermediate mass stars. Therefore the detection of a strong iron deficiency in two PG1159 type central stars was surprising (Miksa et al. 2002).

The central star of the planetary nebula Abell 78 is a very rare [WC]PG1159 transition object showing spectral signatures of both early [WC] spectral type (emission lines) and PG1159 type (absorption lines). The object was well studied in the past, its atmospheric parameters were determined by NLTE model atmosphere analyses (Koesterke \& Werner 1998):

$T_{\text {eff }}=110000 \mathrm{~K}, \log g=5.5, \log \left(\dot{M} / \mathrm{M}_{\odot} \mathrm{yr}^{-1}\right)=-7.3, v_{\infty}=3750 \mathrm{~km} / \mathrm{s}$, $\mathrm{He}=33 \%, \mathrm{C}=50 \%, \mathrm{~N}=2 \%, \mathrm{O}=15 \%$ (by mass).

A high resolution spectrum was taken with FUSE in order to look for iron (Fig. 1). No iron lines are detectable in the observed spectrum. Varying the iron abundance in the NLTE models suggests that the absence of iron lines is due to an underabundance of about two dex, compared to the solar value. For a detailed discussion of possible explanations, e.g. neutron-capture nucleosynthesis, see the respective papers by Herwig et al. and Werner et al. in these proceedings. 


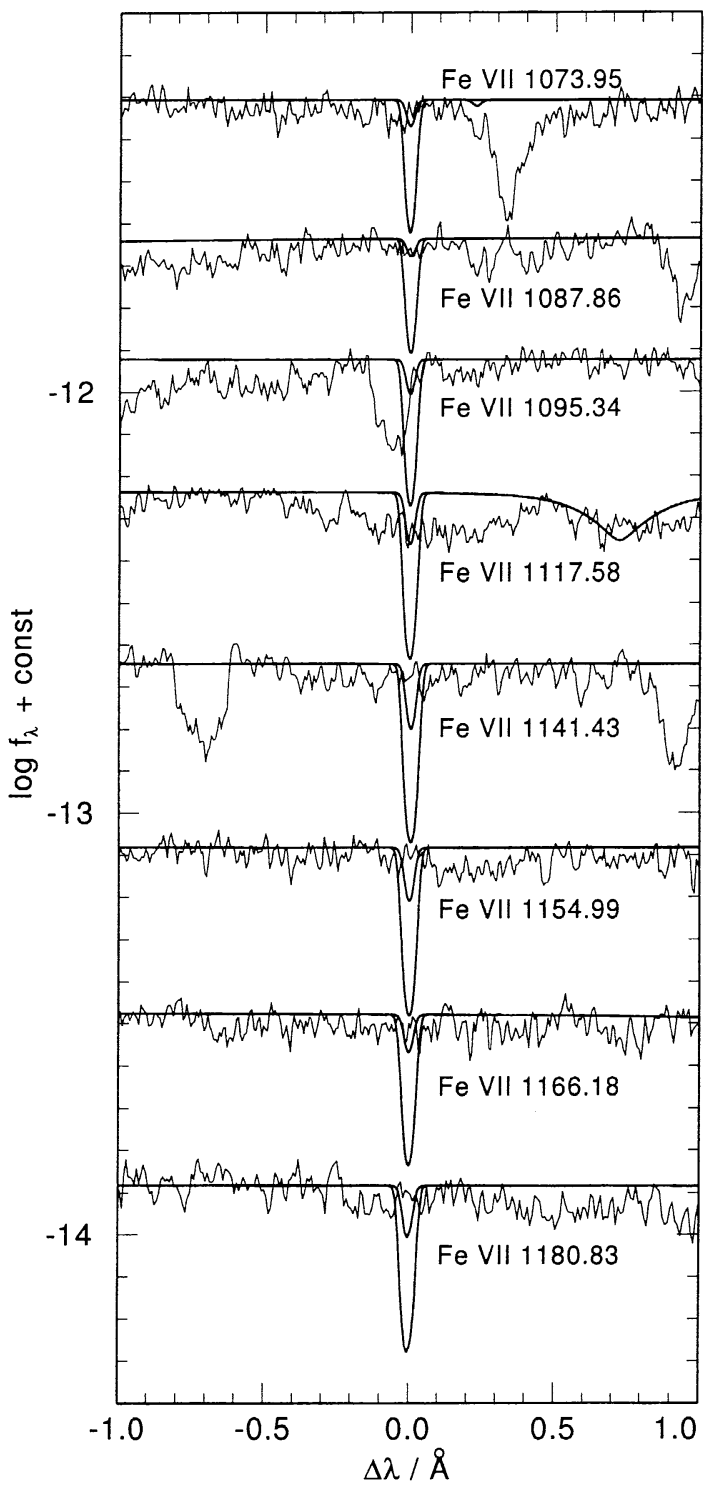

Figure 1. Details of FUSE spectrum of Abell 78 compared to theoretical line profiles, centered around the strongest Fe VII lines in the model. The model iron abundances are 0.1 solar and 0.01 solar. No iron lines are detectable in the FUSE spectrum. The apparent absorption line at $1095.2 \AA$ is a detector artifact.

\section{References}

Koesterke L., \& Werner K. 1998, ApJ 500, L55

Miksa S., Deetjen J.L., Dreizler S., et al. 2002, A\&A, 389, 953 